\title{
A New Watermarking Approach Based on Combination of Reversible Watermarking and CDMA in Spatial and DWT Domain
}

\section{S.Bekkouche}

University of Sciences and Technology Oran USTO Oran, 31100, Algeria chouarfia@univ-usto.dz

\author{
sbekkouche2008@gmail.com
}

\author{
A.Chouarfia \\ University of Sciences and Technology Oran USTO \\ Oran, 31100, Algeria
}

\section{Abstract}

Image watermarking can be defined as a technique that allows insertion of imperceptible and indelible digital data into an image. In addition to its initial application which is the copyright, watermarking can be used in other fields, particularly in the medical field in order to contribute to secure images shared on the network for telemedicine applications. In this report we study some watermarking methods and the comparison result of their combination, the first one is based on the CDMA (Code Division Multiple Access) in DWT(Discrete Wavelet Transform) domain, noted CDMA-DWT and CDMA in spatial domain, noted CDMA-SD and their aim are to verify the image authenticity whereas the second one is the reversible watermarking (the least significant bits LSB and cryptography tools), the reversible carte mapping RCM their objective are to check the integrity of the image and to keep the Confidentiality of the patient data. A new scheme of watermarking is the combination of the reversible watermarking method and the method of CDMA-DWT and the second is the combination of the reversible watermarking and the method of CDMA-sp to verify the three security properties Integrity, Authenticity and Confidentiality of medical data and patient information. In the end, we made a comparison between these methods within the parameters of quality of medical images Initially, an in-depth study on the characteristics of medical images would contribute to improve these methods to measurements have been done on the watermarked image to verify that this technique does not lead to a wrong diagnostic. The robustness of the watermarked images against attacks has been verified on the parameters of PSNR (Peak Signal to Noise Ratio), MSE (Mean Square Error), MAE (Mean Absolute Error) and SNR (Signal to Noise Ratio) which show that the resulting quality of combination watermarking method is good in DWT than other techniques.

Keywords: Watermarking, Reversible watermarking, CDMA-DWT, Integrity, Authentication, Confidentiality. 


\section{INTRODUCTION}

Among the new technologies available to medical informatics some have a secondary impact because they are not specific to the medical field (optical disk, smart card). Others have an impact as it completely changes the use of computers particularly in hospitals. Among these, New network architectures will disrupt the manner to envisage the integration of HIS (Hospital Information Systems) and security of medical information. The diagnosis requires more and more exchanges of medical images from digital modalities and volumes (IRM, X-Scanner, nuclear medicine, etc...) Between public structure of care health facilities (university hospitals or departmental having high debit and allowing several remote experts to issue a notice for a better management of the patient. To stop the copying works of images and contribute to the Copyright Protection., new methods have been developed known more by watermarking.

The watermarking is therefore proposed to ensure greater security by verifying image authentication and integrity, and on the other side, the patient information to be saved or transmitted in a confidential way in medical imagery.

We will focus mainly on the watermarking images and medical data.

Medical imagery is a field where the protection of the integrity and confidentiality of content is a critical issue due to the special characteristics derived from strict ethics, legislative and diagnostic implications. It is very important to prevent unauthorized manipulation and misappropriation of such digitized images. The risks are increased when dealing with an open environment like the internet. Medical images should be kept intact in any circumstance and before any operation they must be checked for:

- Integrity: that is the image or data has not been modified by non authorized people.

- Authentication: that is the image or data belongs indeed to the correct patient.

- Confidentiality: that is protection the medical image and patient information against attacks.

Watermarking is a new technology which hopefully can help in that aim. Before applying watermarking techniques developed for medical imagery applications, it is important that the requirements imposed by medical images are carefully analyzed to investigate whether they are compatible with existing watermarking techniques. Different watermarking techniques have been proposed to address the problems of medical confidentiality protection and both origin and data authentication. In this work, a watermarking technique is adapted to provide the three properties of security authentication, confidentiality and integrity of medical image and patient information. This technique based on combination the CDMA-DWT [1] [10] and the reversible watermarking [2] [9] and. We will give in section 2 an overview of some reversible watermarking techniques and the desired functionalities of watermarking techniques are discussed in terms of medical images. In section 3, the new watermarking approach is presented as a well designed scheme for the medical field. We give the main functionalities of our security system.

Finally, the experimental results are presented to validate the proposed approach.

\section{WATERMARKING TECHNIQUES}

Image watermarking is the process of embedding into image specific information that helps establishing the ownership of the image. Watermarking techniques are divided in two categories.

Spatial Domain Watermarking, where the least significant bits is replaced with watermark, Frequency Domain Watermarking, where the image is first transformed to frequency domain and then the low frequency components are modified to contain the watermark. Watermarking can be applied in frequency domain by applying transforms like Discrete Fourier Transform (DFT) [13], Discrete Cosine Transform (DCT) [7] or the Discrete Wavelet Transform (DWT) [4][12]. Embedding the watermark in the frequency domain can provide more robustness than in the spatial domain. It is strong against attacks like compression where spatial domain is not. Image watermarking techniques can be distinguished according to the way the watermark is revealed 
from the watermarked image. One way is by comparing this image to the original one, while the other doesn't resort to this comparison. The second are usually referred to as blind watermarking techniques and are preferable.

Six different watermarking techniques each from different domain i.e. Spatial Domain and Wavelet Domain [10] watermarking have been chosen for the experiment. The techniques used for the comparative analysis of watermarking process are CDMA in spatial domain noted CDMASD, CDMA-DWT [1] [10], reversible watermarking [3] [10], RCM [8] [11], the reversible watermarking and the proposed approach which is the combination of the CDMA-DWT and the reversible watermarking.

Previous work on embedding invisible watermarks can be broadly grouped into spatial domain and transform domain methods. Typically, the data used to represent the digital watermarks are a very small fraction of the host image data. Such signatures include, for example, pseudo-random numbers, trademark symbols and binary images. CDMA-SD method usually modifies the leastsignificant bits of the host image but the CDMA-DWT technique can be employing to scatter each of the bits randomly throughout the cover image.

$\mathrm{RCM}$ is a simple integer transform that applies to pairs of pixels. For some pairs of pixels, RCM is invertible, even if the least significant bits (LSBs) of the transformed pixels are lost. The data space occupied by the LSBs is suitable for data hiding. The embedded information bit-rates of the proposed spatial domain reversible watermarking scheme are close to the highest bit-rates reported so far. The scheme does not need additional data compression, and, in terms of mathematical complexity, it appears to be the lowest complexity one proposed up to now.

Reversible watermarking (RW) technique which lossless compress the bits to be affected by the embedding operation to preserve the original data and create space for the watermark. The compressed data and the watermark are then embedded into the host image. This practice of compressing original data for reversibility purpose has been widely adopted based on LSB and cryptography selects pixels or transformation coefficients, and then lossless compresses them so as to save space for the watermark. Therefore, it has the property that the embedding distortion can be completely removed from the watermarked image without any side channel. At the other side, the original host image can be recovered in its integrality.

\section{PROPOSED APPROACH}

\subsection{Primary combination}

By applying the reversible watermarking(RW) [2] based on LSB bits and cryptography tools which give an image The method of CDMA-SD [3] is applied in this image for given a watermarked image.

\section{a. Insertion process}

The insertion is the same of that the insertion reversible watermarking process which gives a result, considering this result as a new input or a new original image using the original watermarking dominates the CDMA in spatial domain.

In the insertion process, we scan the image by rows and Lossless compress the bit-stream of LSB values as the image is scanned. Once this compressed bit-stream is obtained, we concatenate it with the encrypted patient information and hash the result of concatenation and embed it into the LSBs by scanning the image in the same pattern. The overall procedure is then a four steps process:

(1) Calculate the authentication code (MAC) of the image Using SHA algorithm [7]

(2) concatenate the authentication code and patient information and encrypt the resulting string;

(3) Select the LSBs of all pixels and compress the resulting string using RLE algorithm [5].

(4) Concatenate the compressed string and the encrypted string and insert them back into the

LSB locations by adding blanks if necessary which give a watermarked image1. 
The insertion process of CDMA in spatial domain

[3] was applied on the watermarked image1 for obtain the watermarked_image.

the insertion primary combination has been shown below in Figure1

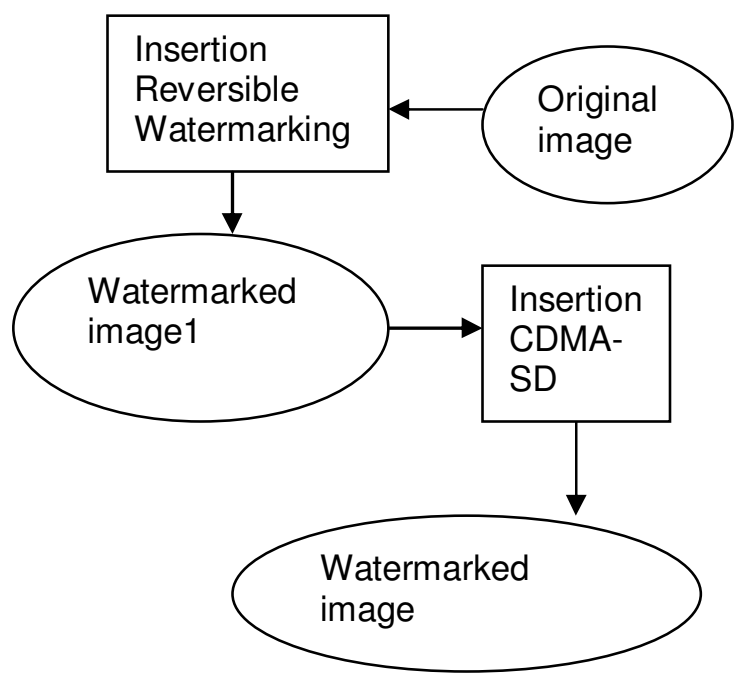

FIGURE1: Insertion primary combination process

\section{b- Extraction process}

- Using the step of the extraction CDMA process [3] on the watermarked image.

- The result will be used as watermarked image1.

- Applying extraction reversible watermarking process [2] on the watermarked image1 to extracting data from LSBs.

- Convert binary to ASCII to get to "@" character represents the end of the data inserted.

- Make a decoding key using the RSA inclusion K.

- Separate the footprint (size unknown) of Patient data and calculate the footprint of image to obtain the original ones.

The extraction primary combination has been shown below in Figure2

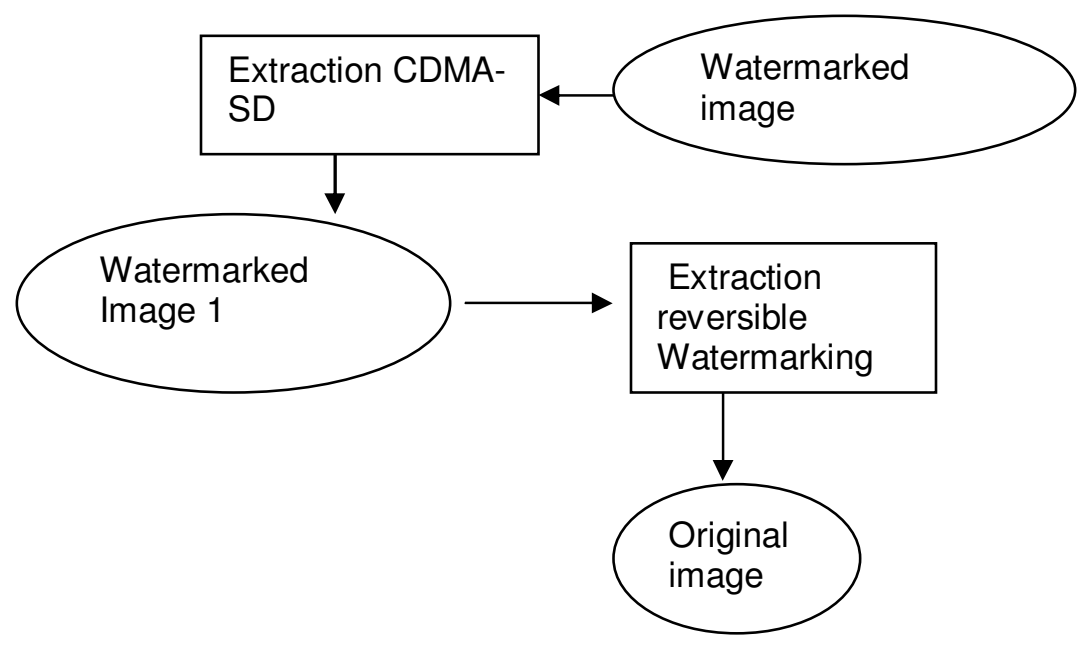

FIGURE2: Extraction primary combination process 


\subsection{Second combination}

By applying the reversible watermarking based on LSB bits and cryptography tools which give an image. The method of CDMA-DWT is applied in this image for given a watermarked image. Then consult the results of insertion and extraction steps and we test the performance against different types of attacks.

\section{a. Insertion process}

By applying the reversible watermarking [2] and CDMA - DWT [1].

1. The insertion process is the same of that of the insertion reversible watermarking process which gives a result.

2. Considering this result as a new input or a new original image using the original watermarking dominates the CDMA in DWT domain.

In the insertion process, we scan the image by rows and Lossless compress the bit-stream of LSB values as the image is scanned. Once this compressed bit-stream is obtained, we concatenate it with the encrypted patient information and the hash the concatenate result and insert it into the LSBs by scanning the image in the same pattern. The overall procedure is then a four steps process:

(1) Calculate the authentication code (MAC) of the image using SHA algorithm [7].

(2) Concatenate the authentication code and patient information and encrypt the resulting string.

(3) Select the LSBs of all pixels and compress the resulting string using RLE algorithm [5].

(4) Concatenate the compressed string and the encrypted string and insert them back into the LSB locations by adding blanks if necessary which give a watermarked image1.

- Generation of the multilayer sequence using a Key K.

- Generation of mark W.

- Decomposition of the watermarked_image1 with a DWT resolution level.

- DWT $(\mathrm{I})=(\mathrm{IA}, \mathrm{DH}, \mathrm{DV}, \mathrm{DD})$

With IA: approximate image $\mathrm{DH}$; horizontal detail, DV and DD vertical and diagonal detail respectively.

- Insertion of the watermark in the three decomposed image details (diagonal, vertical and horizontal).

The mark is weighted by the coefficient $\alpha$.We get the three details scored:

$$
\begin{aligned}
& \mathrm{DH}^{\prime}=\mathrm{DH}+\square \mathrm{W} \\
& \mathrm{DV}^{\prime}=\mathrm{DV}+\square \mathrm{W} \\
& \mathrm{DD}^{\prime}=\mathrm{DD}+\square \mathrm{W}
\end{aligned}
$$

Note that the mark must be the same size as the details.

- Reconstruction of the decomposed image which will give the watermarked image $I^{\prime}$ using the inverse discrete wavelet transforms IDWT:

$$
I^{\prime}=\text { IDWT (IA, DH', DV', DD'). }
$$

The insertion process has been shown below in Figure1. 


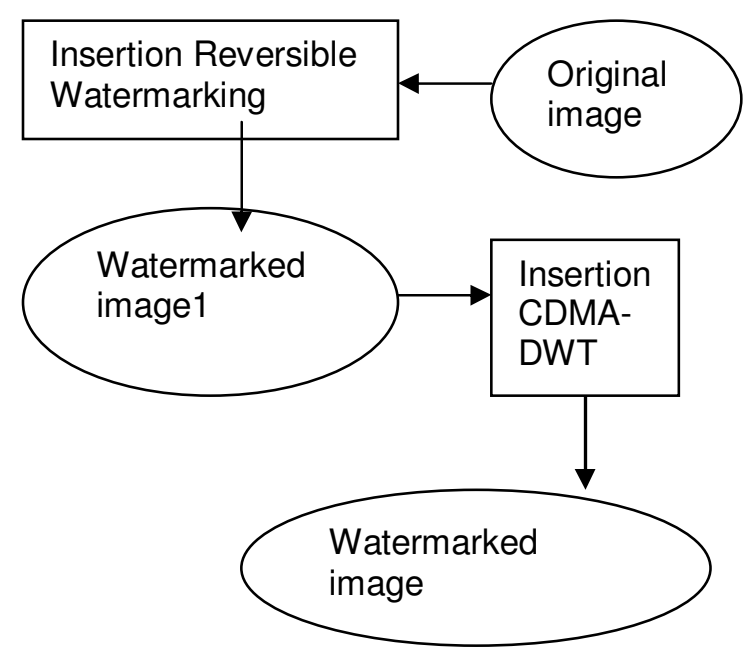

FIGURE1: Insertion second combination process

\section{b. Extraction process}

- Using the extraction steps of the CDMA-DWT technique [1].

- Generation of multilayer sequence with the same key insertion K.

- Decomposition of the image with the DWT into a single resolution level.

- Calculation of the correlation between multilayer sequence and three-layered image detail broken then the message is decoded. According to the sign of the correlation.

The researched data is extracted three times thus allowing us to check and correct them.

- The result will be used as a watermarked Image

- Applying of extraction reversible watermarking method on the image result to get an original image.

The extraction process has been shown below in Figure4

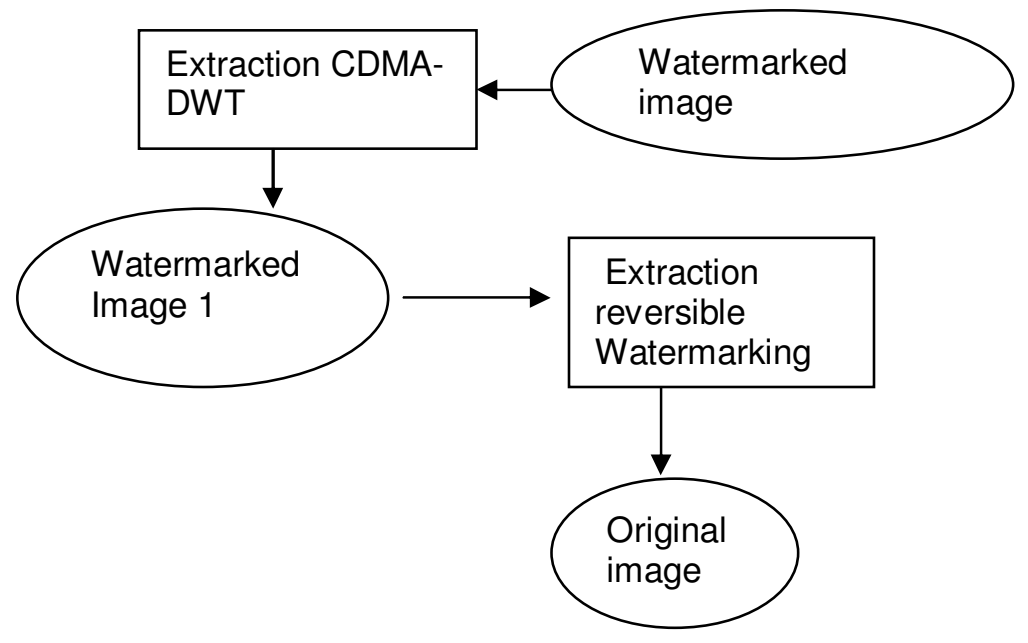

FIGURE2: Extraction second combination process 


\section{IMPLEMENTATION AND RESULT}

\subsection{Test Images}

We have implemented six algorithms (reversible watermarking, CDMA-SD, CDMA-DWT, RCM, combination of reversible watermarking and CDMA-SD and our approach). Based on our proposal to investigate the performance of the algorithms based on PSNR values computed.

The tests were performed on IRM medical images coded on 256 gray levels, bitmap format and size 256x256.

We conducted tests on 10 IRM medical images.

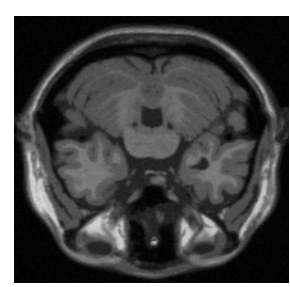

(a)

FIGURE3 : An IRM medical image

\subsection{Insertion and Extraction Data}

To insert the signature, the user must fill out the following input:

- the signature (64 bit)

- The secret key

- the number of layers used

Upon insertion, the user gets the number of bits in the signature. This data is necessary for the detection phase.

To detect the patient data the user must have:

- The marked image

- The key

- The number of embedded bits

After the achievement of six techniques we compare them to evaluate the rate of evaluation of each it.

\subsection{Discussion}

The comparative analysis of the six watermarking techniques has been done on the basis of noise and rotation attacks. Results of the individual watermarking technique have been compared on the basis of PSNR, MSE, MAE and SNR [6] given in Equations (1) to (4).

The obtained PSNRs between original and watermarked images for the six techniques are used to measure the distortion caused by the watermarking. This ratio is often used as a quality measurement between the original and a watermarked image. If the PSNR is higher then the watermarked image quality is better. The PSNR is the square of ratio of maximum pixel value i.e. 255 to the MSE value.

For a good image the SNR value must be high, according to the results established, we select the application field of the watermark where the luminance (Xmax) is maximum. 
The MSE is used to quantify the distortion generated by the digital watermarking. In fact, we use an additive scheme to watermark the image. This modification could hinder the quality of the image. The equation (2) quantifies the mean absolute differences between original image and the watermarked image.

$$
\begin{aligned}
M S E & =\frac{1}{M N} \sum_{i} \sum_{j}\left(I(i, j)-I_{w}(i, j)\right)^{2} \\
M A E & =\frac{1}{M N} \sum_{i} \sum_{j}\left|I(i, j)-I_{w}(i, j)\right| \\
P S N R & =10 \log _{10} \frac{X \max ^{2}}{M S E}=\frac{255^{2}}{M S E} \\
S N R & =\frac{\sum_{1}^{M} \sum_{1}^{N} I_{s}^{2}}{\sum_{1}^{M} \sum_{1}^{N}\left(I_{e}-I_{s}\right)^{2}}
\end{aligned}
$$

\begin{tabular}{|c|c|c|c|c|c|}
\hline & PSNR & SNR & MSE & MAE & $\begin{array}{l}\text { Properties of } \\
\text { Security }\end{array}$ \\
\hline $\begin{array}{c}\text { Reversible } \\
\text { Watermarking (RW) }\end{array}$ & $49.20(\mathrm{~dB})$ & $33.17(\mathrm{~dB})$ & 0.78 & 0.78 & Authentification \\
\hline CDMA-SD & $45.12(\mathrm{~dB})$ & $29.04(\mathrm{~dB})$ & 2.00 & 1.002 & $\begin{array}{l}\text { Integrity and } \\
\text { Confidentiality }\end{array}$ \\
\hline CDMA-DWT & $66.22(\mathrm{~dB})$ & $310.13(\mathrm{~dB})$ & $1.55^{\mathrm{e}-02}$ & $\begin{array}{l}6.75^{\mathrm{e}}- \\
01\end{array}$ & $\begin{array}{l}\text { Integrity and } \\
\text { Confidentiality }\end{array}$ \\
\hline $\mathbf{R C M}$ & $54.84(\mathrm{~dB})$ & $38.71(\mathrm{~dB})$ & 0.213 & 0.213 & Authentification \\
\hline $\begin{array}{l}\text { Combinaison } \\
\text { RW/CDMA-SD }\end{array}$ & $46.51(\mathrm{~dB})$ & $30.44(\mathrm{~dB})$ & 1.45 & 0.228 & $\begin{array}{l}\text { Authentification, } \\
\text { Integrity and } \\
\text { Confidentiality }\end{array}$ \\
\hline $\begin{array}{c}\text { Combinaison } \\
\text { RW/CDMA-DWT }\end{array}$ & $49.17 \mathrm{~dB}$ & 1.265 & 0.7866 & 80.29 & $\begin{array}{l}\text { Authentification, } \\
\text { Integrity and } \\
\text { Confidentiality }\end{array}$ \\
\hline
\end{tabular}

Where $I(i, j)$ is an original image $I_{w}$ is watermarked image, $\mathrm{M} \times \mathrm{N}$ is the image size.

TABLE 1: Comparative parameters of the six techniques.

\subsection{Noise Attacks on Watermarked Image}

The attack methodology on the watermarked image is based on the idea that an attacker does not have any

access to the original image or the watermark image/signature. The attacks are, therefore, done on the watermarked image using only the watermarked image as input. The intruder, i.e. attacker, likely has no idea if the attack worked or not so the results are not known to the attacker.

With addition of salt and pepper noise, performance of watermarking scheme is analyzed. 
- Salt and pepper noise: Figure 4 (d) and figure5 (d), shows the simulation results of watermarked image with salt and pepper noise at a gain factor of 0.02 .

\subsection{Results of Noise Attacks}

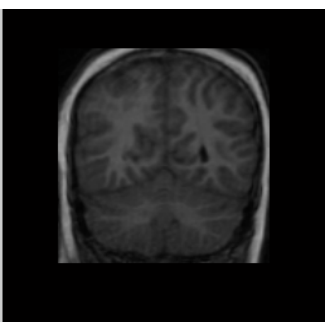

(a)

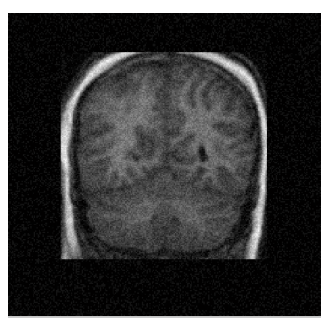

(c)

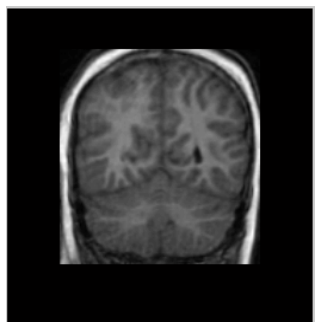

(b)

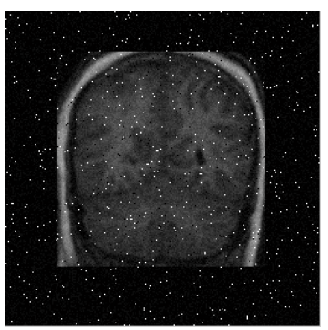

(d)

FIGURE4 : The combination RW/CDMA-SD

(a) Original image,

(b)Watermarked Image after reversible watermarking,

(c) Watermarked Image after RW/CDMA-SD,

(d) Noise watermarked Image

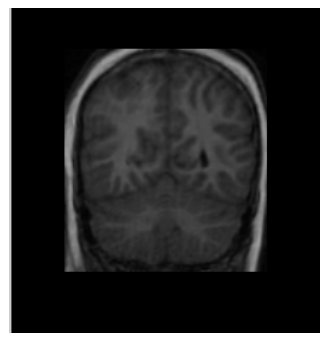

(a)

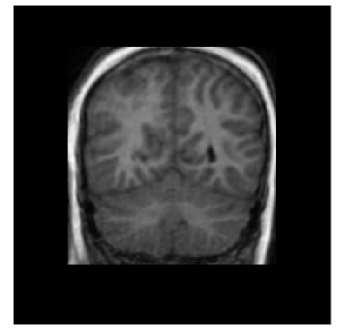

(c)

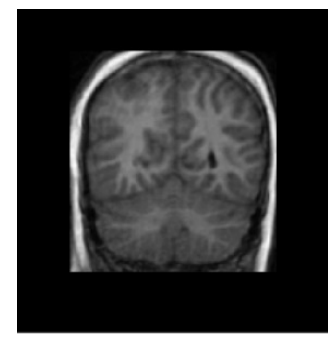

(b)

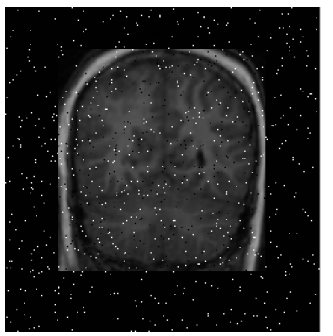

(d)

FIGURE5: The combination RW/CDMA-DWT

(a) Original image,

(b)Watermarked Image after RW, 
(c) Watermarked Image after RW/CDMA-DWT,

(d) Noise watermarked Image

The PSNR have been shown in Table 2 expressed in $(\mathrm{dB})$ is calculated between the original image and noise Watermarked image.

\begin{tabular}{|lcc|}
\hline Method & $\begin{array}{c}\text { PSNR } \\
\text { (dB) }\end{array}$ & MSE \\
\hline $\begin{array}{l}\text { Reversible watermarkiı } \\
\text { (RW) }\end{array}$ & 26.90 & 132.89 \\
RCM & 26.90 & 132.52 \\
$\begin{array}{l}\text { CDMA-SD } \\
\text { in spatial domain }\end{array}$ & $\begin{array}{c}\text { (dB) } \\
\text { CDMA-DWT }\end{array}$ & 146.94 \\
RW/CDMA-SD & 26.7098 & 138.707 \\
Proposed approach & 26.48 & 146.14 \\
\hline
\end{tabular}

TABLE 2 : The Performance analysis of watermarking techniques against noise Attack

\subsection{Results of Rotation Attack}

Rotation attack is among the most popular kinds of geometrical attack on digital multimedia images [8]. Three levels of rotations have been implemented. The original watermarked image is being rotated respectively by 90 degree, 180 degree and 270 degree. The rotation attack has been shown below in Figure 6 .

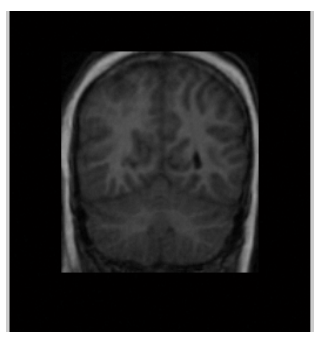

(a)

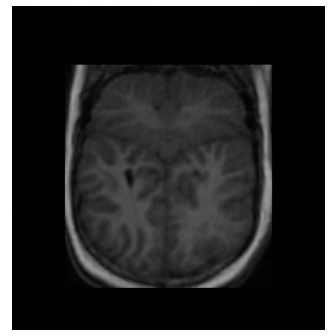

(c)

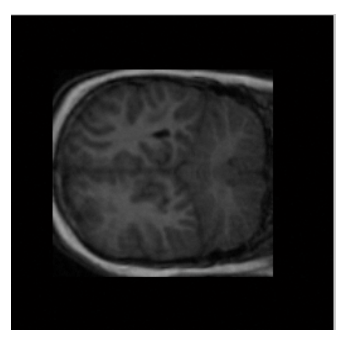

(b)

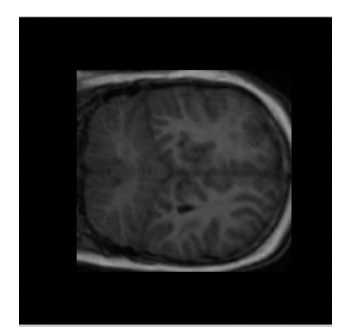

(d)

FIGURE 6 : Rotation attack on the watermarked image

(a) Watermarked image, 
(b) Watermarked image after 90 degree rotation,

(c) Watermarked image after $180^{\circ}$, (d) Watermarked image after $270^{\circ}$.

TABLE 3 : Performance analysis of watermarking techniques against rotation attack

The PSNR values in Table3 show that the combination watermarking in Wavelet domain technique is having the greatest value for the PSNR value. This shows that the wavelet domain watermarking is the best practice for the digital image watermarking purpose.

\section{CONCLUSION}

This paper proposed an efficient digital watermark scheme to increase security, authentication, confidentiality and integrity of medical image and patient information, to transmit it via internet based on combining two watermarking techniques. First technique uses a reversible watermarking by combine the least significant bit and cryptographies tools. Second technique uses the CDMA-DWT. domain.

The watermark can be used to introduce the patient's information in a private and sure manner all while preserving the visual quality of watermarked image.

The experimental results show that our scheme is highly robust against others of image processing operations such as salt and pepper noise. The simulation results show that high quality image i.e. watermarked image with high PSNR is obtained by embedding the watermark in DWT domain than other techniques presented in this article.

The paper focuses on the robustness of the watermarking techniques chosen from all domains of watermarking against rotation attack.

It seems that the proposed approach is best and most robust for medical images watermarking. This work could further be extended to the watermarking purpose of another digital content like audio and video.

\begin{tabular}{|c|c|c|c|}
\hline \multicolumn{2}{|c|}{ Method } & \multirow{2}{*}{$\begin{array}{l}\text { PSNR(dB) } \\
24.5225(\mathrm{~dB})\end{array}$} & \multirow{2}{*}{$\begin{array}{l}\text { MSE } \\
\text { 229.5225 }\end{array}$} \\
\hline $90^{\circ}$ & RW/CDMA-SD & & \\
\hline & $\begin{array}{l}\text { RW/CDMA- } \\
\text { DWT }\end{array}$ & $24.6972(\mathrm{~dB})$ & 220.4724 \\
\hline \multirow[t]{2}{*}{$180^{\circ}$} & RW/CDMA-SD & $25.81 \quad(d B)$ & 170.582 \\
\hline & $\begin{array}{l}\text { RW/CDMA- } \\
\text { DWT }\end{array}$ & $26.083(\mathrm{~dB})$ & 160.225 \\
\hline \multirow[t]{2}{*}{$270^{\circ}$} & RW/CDMA-SD & 24.5225 (dB) & 229.3715 \\
\hline & $\begin{array}{l}\text { RW/CDMA- } \\
\text { DWT }\end{array}$ & $24.6972(\mathrm{~dB})$ & 220.4724 \\
\hline
\end{tabular}




\section{REFERENCES}

[1] Chris Shoemaker, "Hidden Bits: A Survey of Techniques for Digital Watermarking", Independent Study EER-290, 2002.

[2] S. Boucherkha \& M. Benmohamed, A lossless watermarking based authentication system for medical image, In International Journal of Signal Processing, Vol.1, N4, 2004.

[3] B. Vassaux, Technique multicouches pour le tatouage d'images et adaptation aux flux vidéo MPEG-2 et MPEG-4, Thèse de Doctorat, Institut National Polytechnique Grenoble France, 2003.

[4] Y.I. Khamlichi, M. Machkour, K. Afdel, A. Moudden, ' Medical Image Watermarked by Simultaneous Moment Invariants and Content-Based for Privacy and Tamper Detection', Proceedings of the 6th WSEAS International Conference on Multimedia Systems \& Signal Processing, Hangzhou, China, April 16-18, pp109-113, 2006.

[5] Rainer Steinwandt, Viktória I. Villányi, "A one-time signature using run-length encoding", Journal Information processing Letters ,Volume 108 Issue 4,October ,2008.

[6] B. Aiazzi, L. Alparone and S. Baronti. "Near-lossless compression of 3-D optical data". IEEE Transactions on Geosciences and Remote Sensing, vol. 39, no 11, pp: 2547-2557, 2001.

[7] Xu Yan-ping, Jia Li-qin,"Research of a Digital Watermarking Algorithm Based on Discrete Cosine Transform", Proceedings of the Third International Symposium on Electronic Commerce and Security Workshops(ISECS '10) Guangzhou China 29-31 July 2010 pp 373375

[8] Ping Dong, Jovan G. Brankov, Nikolas P. Galatsanos, Yongyi Yang, Franck Davoine, "Digital Watermarking Robust to Geometric Distortions", IEEE Transactions on Image Processing, Vol. 14, NO. 12, December, 2005.

[9] YongJie Wang, Yao Zhao, Jeng-Shyang Pan and ShaoWei Weng,"A Reversible Watermark Scheme Combined with Hash Function and Lossless Compression ", Volume 3682/2005, pp: 1168-1174, DOI: 10.1007/11552451_161, Computer Science, 2005.

[10] Harsh K Verma1, Abhishek Narain Singh, Raman Kumar, «Robustness of the Digital Image Watermarking Techniques against Brightness and Rotation Attack", International Journal of Computer Science and Information Security, Vol. 5, No. 1, 2009.

[11] Yeh-Shun Chen, Ran-Zan Wang, Yeuan-Kuen Lee, Shih-Yu Huang "Steganalysis of Reversible Contrast Mapping Watermarking", Proceedings of the World Congress on Engineering 2008, Vol 1, WCE 2008, July 2 - 4, 2008, London, U.K

[12] W.-T. Huang, S-Y. Tan, Y.-J Chang and C.-H. Chen, "A robust watermarking technique for copyright protection using discrete wavelet transform", WSEAS Trans. Computers, vol. 9, no. 5, pp. 485-495, 2010. 